\title{
A sinonasal yolk sac tumor in an adult
}

\author{
Jaehoon Shin ${ }^{1}$, Ji Heui Kim², Kyeong Cheon Jung ${ }^{3}$, Kyung-Ja Cho ${ }^{1}$ \\ Departments of ${ }^{1}$ Pathology and ${ }^{2}$ Otorhinolaryngology-Head and Neck Surgery, Asan Medical Center, University of Ulsan College of Medicine, Seoul; \\ ${ }^{3}$ Department of Pathology, Seoul National University Hospital, Seoul National University College of Medicine, Seoul, Korea
}

Yolk sac tumors (YSTs), which are also called endodermal sinus tumors, are malignant tumors of germ cell origin. These tumors usually occur in the gonads, but $20 \%$ of cases have been reported at extragonadal sites. The head and neck is a rarely affected region that accounts for just $1 \%$ of all malignant tumors of germ cell origin. In addition, YSTs arise mostly in childhood. We present a rare pathologically pure case of primary adult YST in the sinonasal area. A 45-year-old male patient presented with a rapidly growing mass in the nasal cavity, which caused nasal obstruction and bloody post-nasal drip. The histopathologic features indicated pure YST, and immunohistochemical analysis revealed positive reactivity for Sal-like protein 4 and alpha-fetoprotein. Herein, we discuss the clinical, radiologic, and histologic features of this YST and review other cases of sinonasal YST in adults.

Key Words: Yolk sac tumor; Endodermal sinus tumor; Paranasal sinus; Nasal cavity; Adult

Received: September 6, 2021 Revised: December 7, 2021 Accepted: December 9, 2021

Corresponding Author: Kyung-Ja Cho, MD, PhD, Department of Pathology, Asan Medical Center, University of Ulsan College of Medicine, 88 Olympic-ro 43-gil, Songpa-gu, Seoul 05505, Korea

Tel: +82-2-3010-4545, Fax: +82-2-472-4560, E-mail: kjc@amc.seoul.kr

Yolk sac tumors (YSTs), which are also called endodermal sinus tumors, are malignant tumors of germ cell origin. These tumors usually occur in the gonads, but $20 \%$ of cases have been reported at extragonadal sites. The head and neck is a rarely affected region that accounts for just $1 \%$ of all malignant tumors of germ cell origin [1]. In addition, YSTs arise mostly in childhood. To our knowledge, there are only eight reported cases of sinonasal YSTs in adult patients. We report a very rare case of a pathologically pure form of primary adult YST in the sinonasal area.

\section{CASE REPORT}

A 45-year-old man was referred to the Department of Otorhinolaryngology at Asan Medical Center in Seoul for persistent right nasal obstruction, bloody rhinorrhea, and post-nasal drip. He reported a history of bloody post-nasal drip for the past four months, facial pain for the last three months, visual disturbance in the right lower field, and diplopia. An endoscopic examination showed a 3-cm-sized mass obstructing the right nasal cavity with partially necrotic tissue.

Initial computed tomography and magnetic resonance imag- ing revealed a mass that obstructed the right nasal cavity and caused bone destruction of the bilateral frontal and ethmoidal sinuses with intracranial extension. Destruction of the bilateral superomedial orbital wall and invasion of the right medial orbital wall and right nasolacrimal duct and sac were also identified (Fig. 1).

Endoscopic excisional biopsy was carried out. The specimen was removed in three pieces measuring up to $4 \times 3 \times 1.5 \mathrm{~cm}$, with a pinkish brown color and a fleshy gelatinous texture. The submitted specimen was processed for histopathological examination and revealed typical YST features. The tumor showed mostly reticular and microcystic growth patterns with loose myxoid stroma, but there were also areas of glandular, solid sheet-like, and polyvesicular vitelline patterns. Some SchillerDuval bodies were noted. Intra- and extra-cytoplasmic hyaline globules were identified (Fig. 2). No other tumor components were seen.

Immunohistochemical staining showed diffuse strong nuclear positivity for Sal-like protein 4 and diffuse strong cytoplasmic positivity for $\alpha$-fetoprotein (Fig. 3). P40 was negative, but integrase interactor 1 (INI1) and Brahma-related gene-1 (BRG1) 

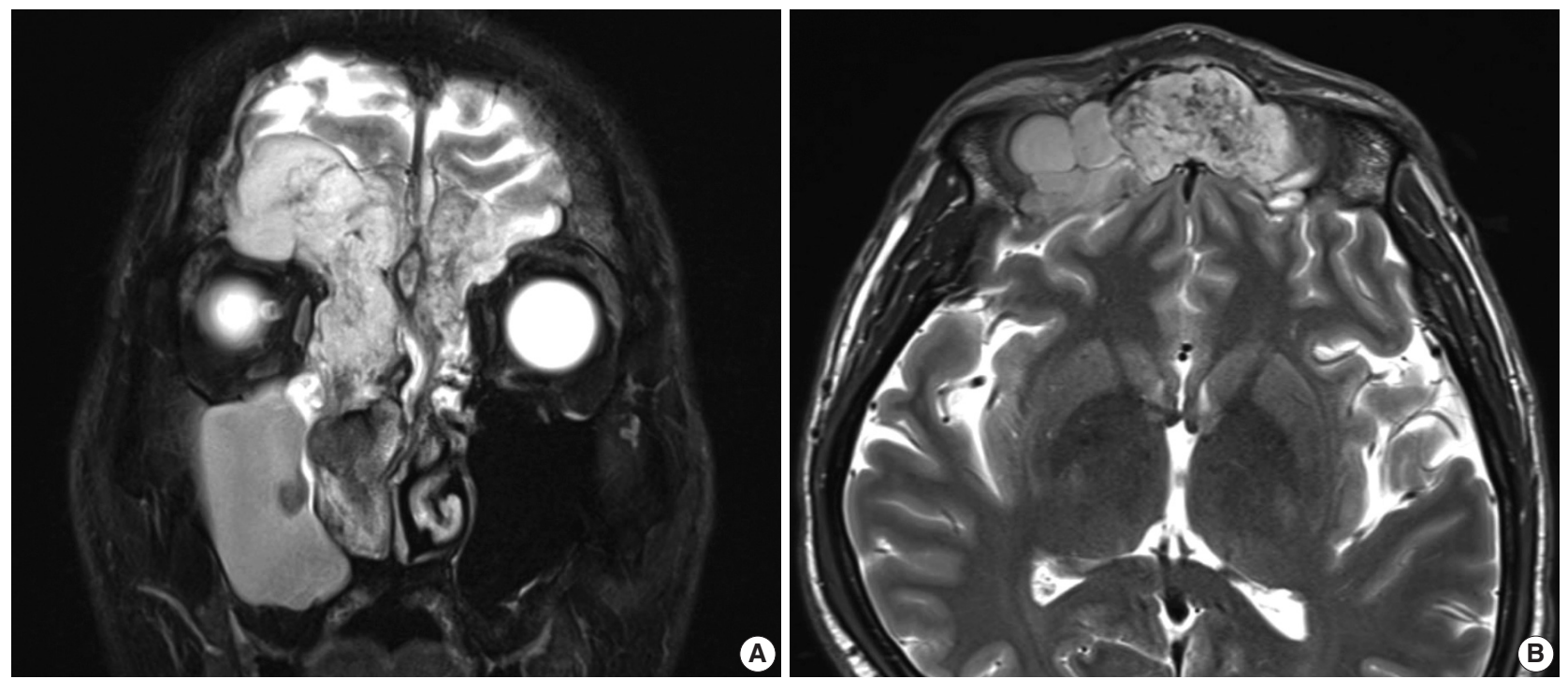

Fig. 1. Coronal (A) and transverse (B) magnetic resonance imaging shows a mass in the right nasal cavity and bilateral frontal sinus.

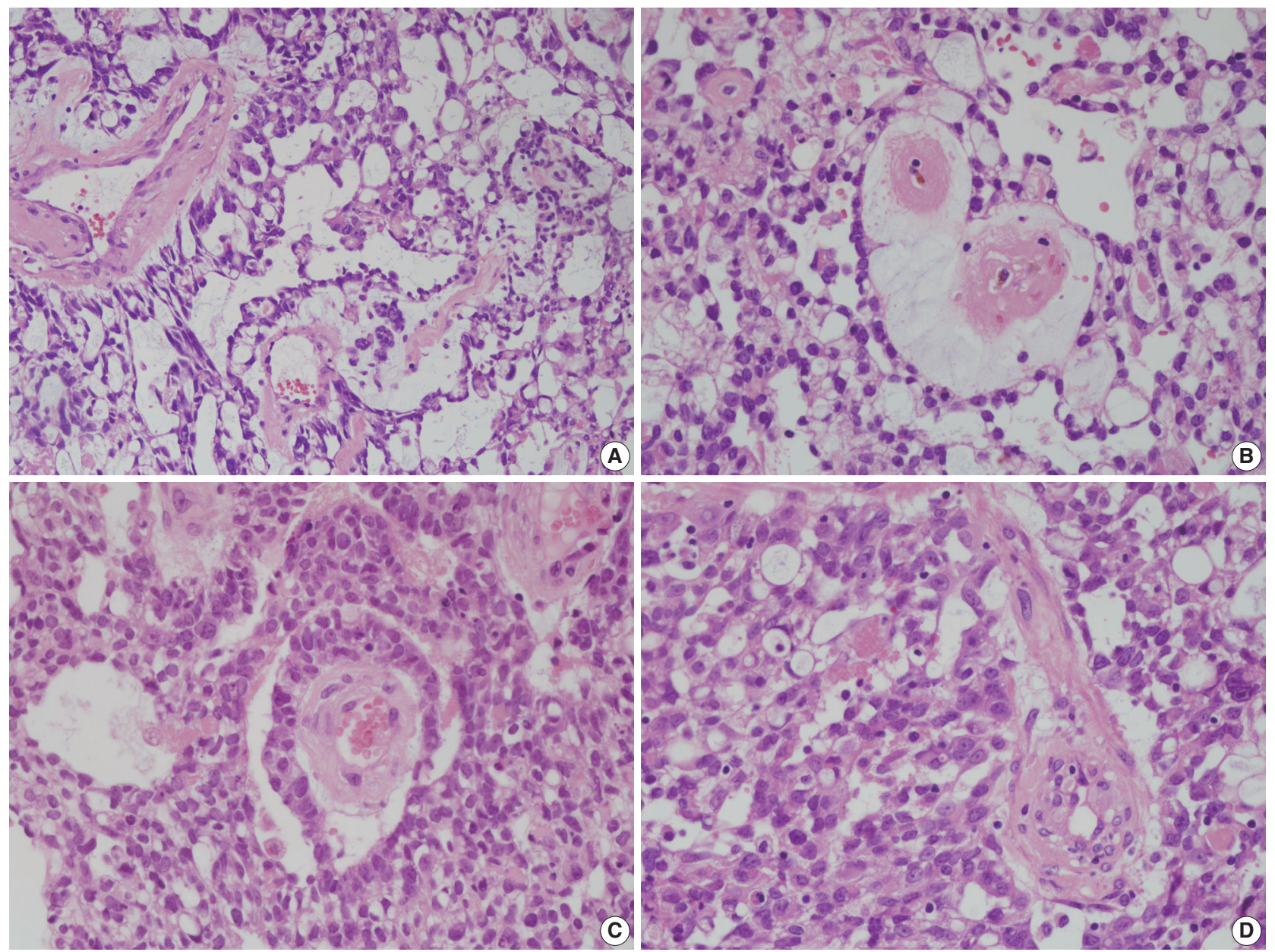

Fig. 2. Histopathologic findings of a sinonasal yolk sac tumor. (A) Reticular and microcystic growth patterns are observed with loose myxoid stroma. (B) A polyvesicular vitelline pattern is occasionally observed. (C) Schiller-Duval bodies are noted focally. (D) Intra- and extra-cytoplasmic hyaline globules are observed. 


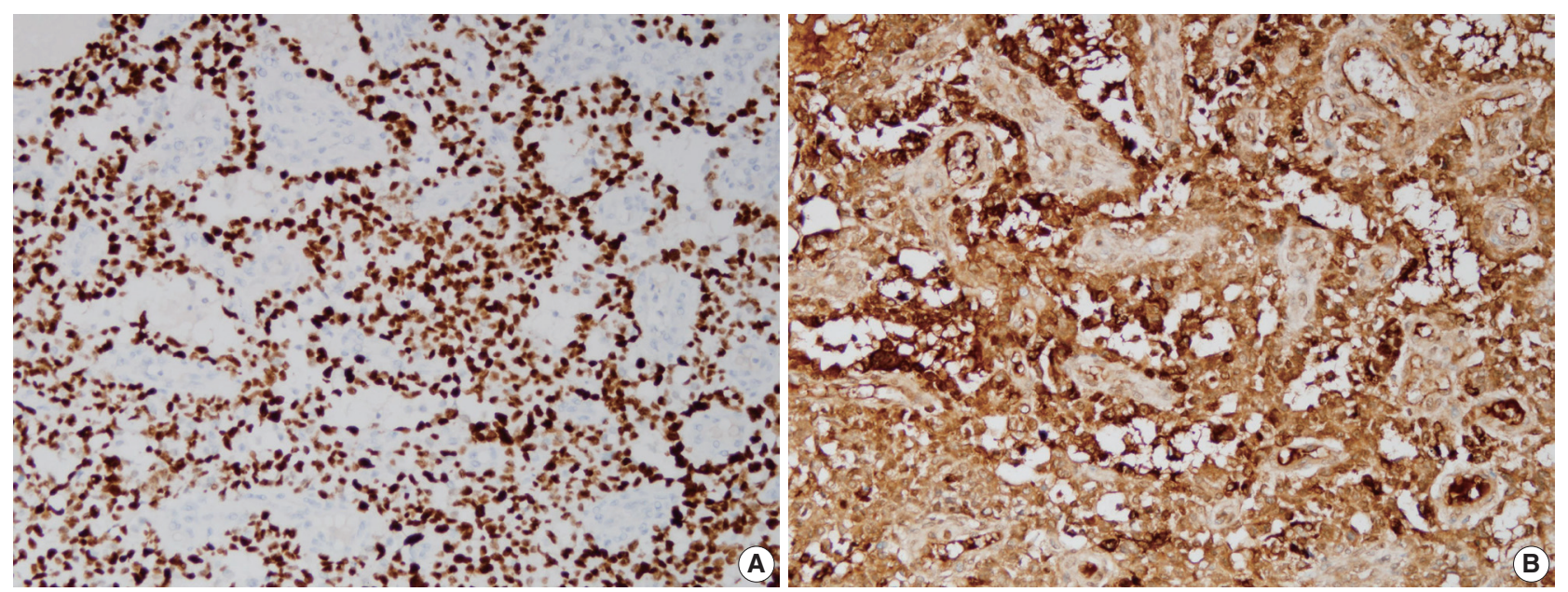

Fig. 3. On immunohistochemical staining, the tumor cells show diffuse strong nuclear positivity for Sal-like protein 4 (A) and diffuse strong cytoplasmic positivity for $\alpha$-fetoprotein (B).
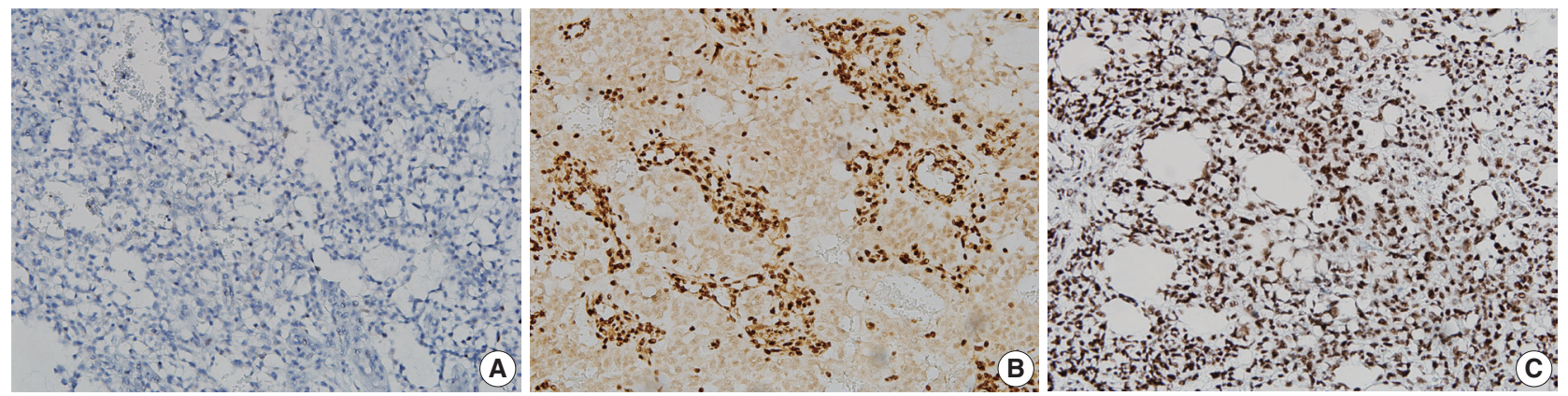

Fig. 4. Immunohistochemical staining revealed that the tumor cells were negative for p40 (A) and diffusely positive for integrase interactor 1 (B) and Brahma-related gene-1 (C).

were both positive (Fig. 4). Therefore, the final diagnosis was pure YST. The patient was started on chemotherapy with a bleomycin, etoposide, and cisplatin regimen and palliative radiation therapy but showed a poor response. He died from the YST at 13 months after diagnosis.

\section{DISCUSSION}

YST usually occurs in the gonads and is primarily found in infants and children. Extragonadal sites have included the sacrococcygeal region, retroperitoneum, mediastinum, brain, and very rarely, the head and neck area [2]. In addition, YST is usually accompanied by other germ cell tumor components, like teratoma or embryonal cell carcinoma. For example, the pure form YST of the testis accounts for just $2.4 \%$ of the reported adult testicular YSTs [3].

Definite diagnosis of YST can be made by biopsy. Upon microscopy, several histological patterns have been described. The most common is a reticular/microcystic pattern, which is a mesh- work of anastomosing spaces and cysts lined by a single layer of tumor cells [4]. Other patterns include endodermal sinus, solid, myxomatous, papillary, glandular, macrocystic, and polyvesicular vitelline [4]. Our case demonstrated reticular, microcystic, and polyvesicular vitelline growth patterns and Schiller-Duval bodies. Shiller-Duval bodies are specific for YST, but they are not essential for diagnosis. The differential diagnoses of this case include sinonasal nonintestinal-type adenocarcinoma, SMARCB1deficient sinonasal carcinoma with pure yolk sac differentiation, and SMARCA4-deficient teratocarcinosarcoma. Clear cytoplasm, an occasional fibrovascular core, and cytokeratin 7 negativity exclude sinonasal nonintestinal-type adenocarcinoma. SMARCB1deficient sinonasal carcinoma usually shows high-grade histologic features, including prominent necrosis and mitoses, but relatively uniform cytology [5]. In our case, INI1 positivity excluded the possibility of SMARCB1-deficient sinonasal carcinoma with pure yolk sac differentiation, and BRG1 positivity ruled out the possibility of SMARCA4-deficient teratocarcinosarcoma.

Eight adult-onset YSTs have been reported in the sinonasal 
Table 1. Previously reported cases of adult-onset yolk sac tumor in the sinonasal area

\begin{tabular}{|c|c|c|c|c|c|}
\hline No. & Age $(y r) / S e x$ & Location & Follow-up & Associated tumor components & Study \\
\hline 1 & $34 / \mathrm{M}$ & Nasopharynx & 1 yr (deceased) & Transitional cell carcinoma & Manivel et al. [6] \\
\hline 2 & $43 / \mathrm{M}$ & Orbit, maxillary, ethmoid and frontal sinuses & $\begin{array}{l}1.5 \text { yr (pulmonary } \\
\text { metastasis) }\end{array}$ & Transitional cell carcinoma & Manivel et al. [6] \\
\hline 3 & $48 / \mathrm{M}$ & Nasal cavity, ethmoid sinus, cribriform plate & 5 yr (disease-free) & Choriocarcinoma & Filho et al. [7] \\
\hline 4 & $59 / \mathrm{M}$ & Orbit, maxillary, ethmoid and frontal sinuses & $1 \mathrm{yr}$ (disease-free) & Poorly differentiated carcinoma & Mishra et al. [8] \\
\hline 5 & $51 / F$ & $\begin{array}{l}\text { Nasal cavity, cribriform plate, anterior cranial fossa, and } \\
\text { frontal sinus }\end{array}$ & - & Teratocarcinosarcoma & Thomas et al. [9] \\
\hline 6 & $58 / F$ & $\begin{array}{l}\text { Nasal cavity, ethmoidal sinus, cribriform plate, and } \\
\text { nasopharynx }\end{array}$ & 0.8 yr (disease-free) & Transitional cell carcinoma & Mei et al. [10] \\
\hline 7 & $44 / F$ & $\begin{array}{l}\text { Nasal cavity, nasopharynx, ethmoid, sphenoid and } \\
\text { maxillary sinuses }\end{array}$ & - & $\begin{array}{l}\text { SMARCB1 (IN11) deficient } \\
\text { carcinoma }\end{array}$ & Zamecnik et al. [11] \\
\hline 8 & $58 / F$ & Nasal cavity, ethmoid sinus, nasopharynx & 3 yr (disease-free) & Transitional cell carcinoma & Wang et al. [12] \\
\hline 9 & $45 / M$ & Nasal cavity, orbit, frontal and ethmoidal sinuses & 13 mo (deceased) & None & Present case \\
\hline
\end{tabular}

$|\mathrm{N}| 1$, integrase interactor 1 .

area in the English literature (Table 1) [6-12]; four cases were accompanied by a transitional cell carcinoma component. Choriocarcinoma, poorly differentiated carcinoma, teratocarcinosarcoma, and SMARCB1 (INI1)-deficient carcinoma components were present in the remaining four cases, respectively. The reason for the common coexistence of a transitional cell carcinoma component in sinonasal YST remains uncertain. In our case, a transitional cell carcinoma component was not observed, as confirmed by $\mathrm{p} 40$ negativity. There are two theories about the origin of extragonadal YST. In the first theory, primordial germ cells are arrested or misplaced during migration from the cranial space to a gonadal site and consequently arrest in the cranial cavity [13]. The second theory holds that extragonadal tumors arise as a result of aberrant somatic differentiation [14]. Previous reports support the second theory. In contrast to previous cases, our case showed histologically pure YST features, which have never been reported. Our case therefore supports the first theory because its histologic features included pure YST not accompanied by other tumor components.

Surgical resection, combined chemotherapy, and radiotherapy are available treatments for YST. Generally, YST is sensitive to chemotherapy. However, the head and neck region, including the sinonasal area as in our case, is very complex and difficult for $\mathrm{R} 0$ resection. As a result, the anatomic location seems to affect the prognosis, although the number of sinonasal YST cases is very small [15]. In addition, increasing age is known to negatively affect clinical behavior, and this trend is consistent with our case findings [16].

In conclusion, although adult-onset sinonasal pure YST is extremely rare, differential diagnosis is important. YST can present with many various scenarios in the sinonasal area, so YST should be considered if the clinical findings are histologically suspicious.

\section{Ethics Statement}

This case was deemed exempt by the Asan Medical Center Institutional Review Board (IRB \#2019-0598). Patient consent waiver was obtained for this study.

\section{Availability of Data and Material}

The datasets generated or analyzed during the study are available from the corresponding author on reasonable request.

\section{Code Availability}

Not applicable.

\section{ORCID}

Jaehoon Shin https://orcid.org/0000-0002-9940-873X

Ji Heui Kim https://orcid.org/0000-0002-9215-6867

Kyeong Cheon Jung https://orcid.org/0000-0002-7741-7184

Kyung-Ja Cho https://orcid.org/0000-0002-4911-7774

\section{Author Contributions}

Conceptualization: KJC, KCJ, JS. Data curation: JHK, KCJ, KJC, JS. Methodology: KJC, JS. Writing_original draft: KJC, JS. Writing_review \& editing: KJC, JS. Approval of final manuscript: all authors.

\section{Conflicts of Interest}

The authors declare that they have no potential conflicts of interest.

\section{Funding Statement}

No funding to declare.

\section{References}

1. Bernbeck B, Schneider DT, Bernbeck B, et al. Germ cell tumors of the head and neck: report from the MAKEI Study Group. Pediatr Blood Cancer 2009; 52: 223-6.

2. Ronchi A, Cozzolino I, Montella M, et al. Extragonadal germ cell tumors: not just a matter of location: a review about clinical, molecular and pathological features. Cancer Med 2019; 8: 6832-40.

3. Khan S, Jetley S, Pujani M, Neogi S. Pure yolk sac tumor of testis in an adult: a rare occurrence. J Postgrad Med 2014; 60: 351-3.

4. Nogales FF, Preda O, Nicolae A. Yolk sac tumours revisited: a review of their many faces and names. Histopathology 2012; 60: 1023-33. 
5. Agaimy A, Hartmann A, Antonescu CR, et al. SMARCB1 (INI-1)deficient sinonasal carcinoma: a series of 39 cases expanding the morphologic and clinicopathologic spectrum of a recently described entity. Am J Surg Pathol 2017; 41: 458-71.

6. Manivel C, Wick MR, Dehner LP. Transitional (cylindric) cell carcinoma with endodermal sinus tumor-like features of the nasopharynx and paranasal sinuses: clinicopathologic and immunohistochemical study of two cases. Arch Pathol Lab Med 1986; 110: 198202.

7. Filho BC, McHugh JB, Carrau RL, Kassam AB. Yolk sac tumor in the nasal cavity. Am J Otolaryngol 2008; 29: 250-4.

8. Mishra A, El-Naggar AK, DeMonte F, Hanna EY. Endodermal sinus tumor of the paranasal sinuses. Head Neck 2008; 30: 539-43.

9. Thomas J, Adegboyega P, Iloabachie K, Mooring JW, Lian T. Sinonasal teratocarcinosarcoma with yolk sac elements: a neoplasm of somatic or germ cell origin? Ann Diagn Pathol 2011; 15: 135-9.

10. Mei X, Xia Y, Sasano H, Gao H. Sinonasal yolk sac (endodermal sinus) tumor in an adult female: a case report and review of the literature. APMIS 2015; 123: 810-4.
11. Zamecnik M, Rychnovsky J, Syrovatka J. Sinonasal SMARCB1 (INI1) deficient carcinoma with yolk sac tumor differentiation: report of a case and comparison with INI1 expression in gonadal germ cell tumors. Int J Surg Pathol 2018; 26: 245-9.

12. Wang P, Hou G, Li F, Cheng X. A case of primary sinonasal yolk sac tumor. Clin Nucl Med 2020; 45: 908-9.

13. Oosterhuis JW, Looijenga LH. Human germ cell tumours from a developmental perspective. Nat Rev Cancer 2019; 19: 522-37.

14. Hodgson A, Ghorab Z, Parra-Herran C. Somatically derived yolk sac tumor of the ovary in a young woman. Int J Gynecol Pathol 2021; 40: 296-300.

15. Heerema-McKenney A, Harrison MR, Bratton B, Farrell J, Zaloudek C. Congenital teratoma: a clinicopathologic study of 22 fetal and neonatal tumors. Am J Surg Pathol 2005; 29: 29-38.

16. McKenney JK, Heerema-McKenney A, Rouse RV. Extragonadal germ cell tumors: a review with emphasis on pathologic features, clinical prognostic variables, and differential diagnostic considerations. Adv Anat Pathol 2007; 14: 69-92. 